\title{
Linx
}

Revue des linguistes de l'université Paris X Nanterre

40 | 1999

Le statut d'unité lexicale

\section{La double hybridation de l'unité lexicale}

\section{Gérard Petit}

\section{OpenEdition}

\section{Journals}

Édition électronique

URL : http://journals.openedition.org/linx/780

DOI : $10.4000 / \operatorname{lin} x .780$

ISSN : 2118-9692

\section{Éditeur}

Presses universitaires de Paris Nanterre

\section{Édition imprimée}

Date de publication : 1 juin 1999

Pagination : 137-158

ISSN : 0246-8743

\section{Référence électronique}

Gérard Petit, «La double hybridation de l'unité lexicale », Linx [En ligne], 40 | 1999, mis en ligne le 22 juin 2012, consulté le 19 avril 2019. URL : http://journals.openedition.org/linx/780 ; DOI : 10.4000/ $\operatorname{lin} x .780$

Ce document a été généré automatiquement le 19 avril 2019.

Département de Sciences du langage, Université Paris Ouest 


\title{
La double hybridation de l'unité lexicale
}

\author{
Gérard Petit
}

1 Le statut d'unité lexicale, tel qu'il est traditionnellement pensé par la lexicologie, produit une schématisation monolithique de son objet. Les modèles de description classiques et fondateurs (retenons essentiellement ceux développés par Saussure, Pierce, Culioli et Mel'Cuk), mais également des alternatives récentes (sémantique du prototype (Rosch, in Kleiber, 1990), clivage Propriétés Extrinsèques / Propriétés Intrinsèques (Cadiot, 1997 ; Cadiot et Nemo, 1998), topoï (notamment Anscombre (1995)) sont lestées par le présupposé que la structure sémantique et référentielle de l'unité lexicale (désormais UL) est intrinsèquement homogène ${ }^{1}$ : elle serait constituée en une totalité stabilisée par la complémentarité sémiotique de ses constituants, que ceux-ci soient des traits exprimant des propriétés, des rubriques qualifiant des fonctions (p.ex. dans la théorie Sens-Texte) ou des faisceaux d'opérations portant sur des repères. D'une manière analogue si le mode de référenciation de l'UL est interrogé (surtout par la perspective culiolienne et la praxématique), l'investigation ne va pas jusqu'à remettre en cause les conditions propres de son effectuation et à demander si les unités d'une même catégorie syntaxique réfèrent toutes lexicalement au même titre.

2 D'où la sélection de corpus non disruptifs et tout à fait adaptés à une modélisation fermée sur le signe: unités ou emplois appartenant à un registre unique (massivement standard) ; signification représentable au moyen d'une matrice unaire (formalisation en sémème, en schème ou gestalt) ; fonction référentielle dénominative ou assimilée.

3 De fait, les généralisations produites sur ce matériau ont conduit à véhiculer une représentation uniformisée du lexique, et à légitimer par là même l'occultation de l'étude de pans entiers de celui-ci (termes familiers, noms de marques déposées, realias enregistrées par les dictionnaires, noms de fêtes religieuses...) les laissant pour compte, ou au mieux les intégrant à titre d'aporie (Martin 1976).

4 Nous plaiderons ici pour une approche différente du statut d'unité lexicale et envisagerons l'UL comme une structure dont la sémiotique est traversée d'hétérogénéité, c'est-à-dire d'hybridation (Petit 1998a et 1999). De fait, si une modélisation est possible, elle se pense comme ouverte et considère l'UL comme un espace composite qui tire son 
identité de sa capacité à générer de la diffraction. La première partie de cette étude sera consacrée à la dimension interne de l'hybridation, qui intéresse la constitution même du signifié lexical et engendre sa stratification. La seconde partie abordera la dimension externe, informée par le mode de référenciation des items. Les observations s'appuieront d'abord sur un corpus d'items considérés traditionnellement comme atypiques par la lexicologie : des noms familiers. Elles seront ensuite étendues aux items jugés typiques.

\section{L'hybridation sémiotique interne et la stratification du signifié lexical}

\section{a. le signifié mémoriel}

5 Nous prendrons ici l'exemple des noms familiers (désormais $\mathrm{N}$ FAM) ${ }^{2}$. Traditionnellement, la signification des N FAM est perçue comme une structure uniforme : les dictionnaires en rendent compte par une périphrase de type logique, un renvoi à un synonyme standard ou à une base de dérivation :

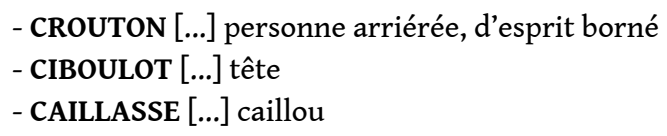

6 De la même manière sur le plan sémantique (Martin 1976) la signification d'un N FAM ( bouquin) se résumerait à une formule sémique identique à celle du $\mathrm{N}$ standard auquel il correspond (livre).

7 À l'inverse de ces hypothèses, nous avons démontré dans Petit (1998a) que la structure du signifié de certains noms familiers comme cigare (" tête, crâne »), citron (idem), citrouille (idem), carafe (idem), caillou (idem), crinière («chevelure abondante») ou cadavre ( «bouteille d'alcool bue entièrement») est stratifiée. Elle est partagée entre ce que l'on peut considérer comme un signifié référentiel, puisqu'il est vrai que :

- cigare, citron, citrouille, carafe signifient, dans un de leurs emplois lexicalisés, «tête,

crâne»

- crinière signifie, dans un de ses emplois lexicalisés, «chevelure abondante "

- cadavre signifie, dans un de ses emplois lexicalisés, «bouteille d'alcool bue entièrement »

8 La signification lexicale du N FAM comprend également ce que nous avons appelé un signifié mémoriel. L'emploi familier de ces $\mathrm{N}$ n'est pas disjoint des autres valeurs constitutives de leur polysémie et notamment de l'emploi de base à partir duquel l'acception familière à été issue par métaphore, métonymie ou autre procédé sémantique (p.ex. pour citron il s'agit de l'emploi « fruit», pour caillou de celui relatif au fragment de pierre, pour cadavre de " corps mort, surtout en parlant de l'homme et des gros animaux » etc.).

9 Le signifié mémoriel est une composante du contenu sémantique global de l'item. Il diffère du signifié référentiel dans la mesure où sa fonction première n'est pas de viser le référent ou d'en assurer l'identification, la communication etc. Il en est incapable. Le signifié mémoriel prend en charge, au sein d'un emploi référentiel dérivé et lexicalisé, c'est-à-dire stable et récurrent (citron dans sa valeur "tête») la réminiscence des conditions sémantiques d'élaboration de celui-ci (citron dans sa valeur « fruit»).

L'emploi familier d'un N polysémique (citron: " tête ») résulte de la rencontre et de deux formules sémantiques hétérogènes («fruit " et «tête»), associées à des référents 
différents ${ }^{3}$. Cette rencontre est rendue possible par la présence de connexions sémantiques plus ou moins lâches entre celles-ci. Le signifié mémoriel manifeste, à la manière d'une mémoire, l'existence de ces connexions et les met en perspective par une figuration.

Le signifié référentiel de ces $\mathrm{N}$ familiers est le site d'attraction et d'accueil d'un signifié hétérogène à celui qui fonde l'emploi de base en standard (p.ex. pour citron « tête, crâne " relativement à «fruit »). Du fait de la plasticité variable des connexions sémantiques, la prédictibilité des emplois FAM reste très aléatoire, bien que le paradigme des reformulants soit régulé par un jeu de matrices (Petit 1998a). Parfois elle est nulle, comme dans le cas de cigare " rouleau de tabac » vs " tête ». Il existe néanmoins un recouvrement sémantique entre les deux, même si la diachronie en a effacé la trace (c'est le cas pour cigare ou caboche).

Si le signifié référentiel assure la dénotation, la signification lexicale (c'est lui qui est convoqué par la question Que signifie $N$ ?, c'est également lui que les dictionnaires enregistrent et qui a pour fonction de viser le référent concerné par l'emploi familier du $\mathrm{N})$, le signifié mémoriel est le lieu du sens comme figuration et déplacement. Il est le site d'élaboration d'un tableau, au sens frégéen du terme, du référent. Si caillou, citron et cigare sont synonymes référentiellement (ils signifient «tête, crâne »), ils ne le sont pas sur le plan mémoriel. L'un conceptualise son référent comme un fragment de pierre, l'autre comme un fruit, le troisième comme un petit rouleau de tabac. Cette conceptualisation joue pour le locuteur un rôle actif lors de la sélection de l'item à employer à l'intérieur du paradigme des reformulants familiers disponibles dans le lexique pour un même $\mathrm{N}$ standard. La configuration du tableau, la nouvelle gestalt que produira le N FAM dans l'interlocution sont prises en compte activement dans le processus de nomination.

Ces deux signifiés interfèrent :

- au sein du N FAM le signifié mémoriel agit sur le signifié référentiel. Il exprime, en le mettant en perspective, ce qui, à l'intérieur ou à l'arrière-plan du signifié référentiel fait sens (et que nous appellerons au paragraphe suivant le signifié topique). Dans les emplois familiers de citron et carafe le signifié mémoriel sélectionne au sein du signifié référentiel « tête, crâne » les traits de sphéricité, de contenance et, pour le second $\mathrm{N}$ celui de rigidité ainsi que l'opposition plein / vide. De même le signifié mémoriel de crinière, caserne, caisse (1. "poitrine»; 2. "voiture») sélectionne dans son homologue référentiel des informations relatives à la taille et la texture (crinière) à la socialité (caserne), à la contenance, au volume (caisse au sens 1) à la fonctionnalité (caisse au sens 2).

Pareillement, le signifié référentiel du N FAM construit les arguments de l'expression du sens. Le signifié référentiel de l'emploi FAM (ici «tête») sélectionne les éléments qui, dans le signifié référentiel de base du même $\mathrm{N}$ («fruit» de citron) produiront par déplacement la figuration du sens (forme du fruit, texture, contenance, fonctionnalité).

- entre le N FAM et son homologue standard. Deux cas de figures sont envisageables. Soit le signifié référentiel du N FAM est identique au signifié du N standard correspondant ( citron, cigare, caisse), soit le N FAM apporte une spécification au N standard (crinière « chevelure abondante »-, poubelle - « voiture sale, mal entretenue »). Dans ce dernier cas le signifié référentiel familier exprime et construit les conditions de la normalité du référent en standard. Une chevelure doit être de densité moyenne, une voiture doit être entretenue, etc. 
17 Dans le cas de citron, cigare ou caisse, le signifié référentiel du N FAM n'apporte aucune spécification relativement à celui de tête. C'est le signifié mémoriel qui construira ici la normalité du référent en standard tout en proposant un déplacement métaphorique de celle-ci. La tête se doit en principe d'être rigide, sphérique et susceptible ou non de se vider ou d'être vidée (au sens métaphorique du terme).

De la même manière, le signifié de tête sélectionne à l'intérieur du signifié référentiel de citron et carafe (dans leur emploi standard) les instructions relatives au caractère massif du fruit, à la pratique qui consiste à en exprimer le jus, à la valeur de récipient rigide de la carafe comme conditions d'une pertinence lexicale.

Qu'il soit mémoriel ou référentiel, le signifié stratifié du nom familier n'est pas une structure statique, un concept modélisable par un listage fixe et purement informationnel de traits, mais un hybride dynamique, et ce à double titre :

- il ouvre en permanence le signe familier sur son extérieur lexical

- il opère un travail sur la signification non familière de cet extérieur et manifeste ce qui en elle fait sens.

Deux catégories d'UL ont été laissées jusqu'ici en suspens, les reformulants familiers monosémiques et les $\mathrm{N}$ polycatégoriels. Les premiers ne sont pas, à l'instar des autres $\mathrm{N}$ FAM entrevus précédemment, des instances de régulation lexicale du sens standard. Du fait de leur structure sémantique, le signifié mémoriel est a priori vide (caboche, clebs, cagibi). Aucune trace d'accrochage sémantique n'est effectivement décelable en synchronie. Dans ce cas un investissement sémantique est toujours possible (Petit 1998a). Toutefois ici la figuration ne sera pas produite par la langue mais individuellement par le locuteur, donc de manière plus ou moins incontrôlable (Nicolas 1995). Il relèvera de la connotation au sens de Martin (1976). Par voie de conséquence ces N ne prennent pas en charge non plus l'expression lexicale du signifié topique du $\mathrm{N}$ standard auquel ils correspondent. En revanche, s'ils manifestent l'expression d'un sens topique, ce dernier ne peut être lexical mais individuel et subjectif, donc relevant lui aussi de la connotation. Les valeurs de sens dont caboche est investi dans l'univers de croyance du locuteur sont analogues à et pointent celles de tête à l'intérieur du même univers de croyance individuel.

21 Les N FAM polycatégoriels sont constitués d'unités qui disposent de plus d'un équivalent standard. Ils réfèrent de manière transversale du fait qu'ils recouvrent des catégories disjointes. Ainsi merde, hormis son emploi «excrément » peut se dire aussi bien d'un humain que d'une chose :

- tiens, reprends ta merde (ta merde : tes affaires, ton livre...)

- il m'a fait une merde (une merde : une mauvaise action...)

- je ne parle pas à une merde (une merde : qqn que l'on méprise)

Le N FAM assume son emploi référentiel (ici « tout $\mathrm{x}$ considéré comme extrêmement méprisable ») à un niveau hiérarchiquement plus élevé que celui de son acception de base (" excrément»). Le degré de généralisation visé le conduit à s'appliquer à plus d'une catégorie référentielle, contrairement aux items observés jusqu'ici. Il n'est également le pendant familier d'aucun $\mathrm{N}$ standard particulier mais plutôt le quasi-hyponyme du $\mathrm{N}$ standard qui couvre le noeud hiérarchique subsumant la diversité référentielle à laquelle il s'applique (en l'occurrence ici le N chose). Malgré cette spécificité, le N FAM dispose effectivement d'un signifié mémoriel, qui construit une figuration du référent, ici rapporté à un excrément. 
Ce genre de N FAM exprime, comme les précédents, les conditions de la normalité du référent couvert par le $\mathrm{N}$ standard. Il induit, dans le cas de merde, des informations sur la qualité et l'intégrité du $\mathrm{x}$. Toutefois, vu le degré de généralité auquel s'opère la visée référentielle, celles-ci ont valeur d'universaux (tout $\mathrm{x}$ est a priori respectable) pour les $\mathrm{N}$ standards hyponymes de celui dans lequel elles sont mises en perspectives par la reformulation en registre familier (ici affaires, livre, action, personne...).

\section{b. le signifié topique}

Jusqu'à présent nous avons (presque) considéré que seul le signifié du N FAM était stratifié et incluait une composante manifestant le sens d'un item par delà sa signification référentielle. Il convient toutefois d'étendre l'observation.

Par un retour de perspective, le lexème familier travaille le sens (et non la signification) du lexème standard et révèle, là où on ne l'attendait peut-être pas, l'hybridation sémantique de celui-ci. Tête, dans son emploi " partie supérieure du corps de l'homme... », ou citron dans son acception « fruit » disposent d'un signifié référentiel, exprimable par un faisceau de traits comme la pratique en est adoptée traditionnellement par la lexicologie. Une approche prototypique (Kleiber 1990) ou par graphe connexe (Grunig B.N. 1982, Grunig B.-N. et R.1985) indiquerait que certains font davantage sens que d'autres. Plutôt que d'envisager une gradation d'indice entre les différentes instructions qui constituent le signifié référentiel standard, l'existence de paradigmes de reformulants familiers lexicalisés (celui de tête " crâne » est constitué de citron, citrouille, carafe, carafon, caillou, coloquinte, ciboulot, caboche, cloche, cigare) tend à démontrer que les items standards présentent également une stratification de leur signifié, selon des paramètres fonctionnellement analogues à ceux des N FAM : le contenu d'un item est réparti entre une signification (dénotative, référentielle) et un sens, que nous appellerons le signifié topique $e^{4}$. La construction et l'expression de ce dernier n'est pas prise en charge par un faisceau mémoriel, mais autonomisé à l'extérieur du $\mathrm{N}$, dans un paradigme de reformulants. Ce signifié, du fait de sa lexicalisation n'est pas ou peu susceptible d'évolution.

Le signifié d'un $\mathrm{N}$ standard est donc lui aussi stratifié, constitué d'une signification dénotative dont la représentation dépendra, pour le linguiste, du modèle de description adopté $^{5}$, et d'un contenu topique dont la trace est attestée par l'ensemble des reformulants lexicaux et lexicalisés que l'on peut recenser autour de l'item. Ce signifié topique est lui aussi lexicalisé, socialisé. Il est constitué d'un faisceau d'informations dont une partie est originellement non indexée. Chaque reformulant instancie ce faisceau en actualisant (la mise en binôme d'un item avec un NFAM constitue un contexte d'actualisation, le reformulant étant un prédicat appliqué au reformulé) l'une ou l'autre, ou la totalité, de ces instructions. Il vectorialise positivement ou négativement celles qui en entrée sont non-indexées : l'instruction de masse (plein vs creux) n'est pas marquée dans le signifié topique de tête mais est indexée [+] par citron et caillou et [-] par cafetière et carafe.

27 Ainsi, concernant tête, les informations suivantes semblent pertinentes: forme du référent, consistance, masse, dimensions, autonomie, fonction. La fonction du crâne appartient au stéréotype courant de la tête et est décrite par les dictionnaires les plus courants (contenir le cerveau...). Elle apparaît donc comme a priori indexée (la fonction est celle de contenant; le contenu est le cerveau) à l'intérieur du signifié topique. En 
revanche ce qui a trait à la forme du référent, sa consistance, son caractère massique, ses dimensions, son autonomie restent non indexées puisqu'elles peuvent faire l'objet de négociations divergentes, voire opposées par le paradigme des reformulants. Les $\mathrm{N}$ d'espèces végétales et minérales (citron, citrouille, caillou...) envisagent la tête comme massique, à l'inverse des $\mathrm{N}$ de produits manufacturés (carafe, cloche) qui la voient creuse. Le même partage concerne l'attribution de la consistance (dure et cassante pour les $\mathrm{N}$ de produits manufacturés et caillou; plutôt souple pour les $\mathrm{N}$ de végétaux). Certains $\mathrm{N}$ comme cloche, citrouille, coloquinte, cassis, citron envisagent le référent comme un tout autonome, non rattaché, alors que d'autres (cigare, ciboulot) ne le décrivent que comme extrémité. Enfin si citrouille et coloquinte s'appuient sur une amplification de la dimension du référent, cassis et caillou produisent une minoration.

Ce signifié topique appartient à la structure sémantique de l'item standard non pas comme un surplus (c'est ce qui le différencie d'une connotation), mais comme une sélection d'instructions appartenant au signifié référentiel et sur l'extension duquel il renseigne. Si l'on considère que la référenciation par nomination est la condensation d'une prédication (tête pour "partie supérieure du corps de l'homme...»), le sens d'un item standard semble receler des informations qu'une formule sémantique, du type sémique, prototypique ou un schéma d'opérations, ne prendrait probablement pas en compte (le caractère massique, les dimensions) ou très partiellement (à l'inverse de la fonction, l'autonomie et la forme du référent ne sont abordées qu'aléatoirement par les dictionnaires) du fait de la limitation quantitative qui lui est généralement imposée mais surtout du fait que cette formule est incapable d'instancier des informations à indexation variable.

Les instructions que contient le signifié topique semblent confinées, dans le processus de condensation que constitue la nomination lexématique (Kleiber 1984), pour partie (les instructions non-indexées) sinon dans l'implicite du moins dans un arrière-plan de la signification, l'inconscient du signe linguistique. Cet inconscient est travaillé de deux manières :

- d'abord dirons-nous par exhumation (au sens étymologique du terme). Étant révélé par d'autres items lexicaux, il n'est pas immédiatement accessible à l'analyse (raison pour laquelle une modélisation fermée sur le signe reste stérile à le décrire et même simplement à envisager son existence). La reformulation révèle, par lambeaux, un faisceau dont l'extension complète n'est à rechercher que dans un paradigme lexicalisé exhaustif ;

- en l'indexant individuellement et parfois différentiellement, les reformulants actualisent ce qui n'est que virtuel mais en instance et appel d'actualisation. Ce contenu signifiant présente en commun avec l'inconscient une forme d'insistance à percer derrière l'explicite et l'évidence.

Des questions restent posées quant à l'hybridation de certaines catégories d'UL. À commencer par les lexèmes standards qui ne disposent pas de reformulant familier attesté dans le lexique ou d'un reformulant dont le signifié mémoriel est a priori vide (piaf pour oiseau, clebs, clébard, cabot pour chien). Si l'unité appartient à une strate inférieure de la hiérarchie lexicale, l'hyperonyme et les différents superordonnés viendront instancier la fraction du signifié référentiel qui fait sens et qui, dans une définition logique, occupe 
la fonction d'incluant. Dans ce cas le signifié topique se distingue de ce que nous avons vu précédemment en ce que :

- il est d'entrée de jeu vectorialisé : les instructions ne sont pas contenues comme a priori nonindexées. Elles sont déjà indexées parce qu'extraites du signifié dénotatif et que leur indice n'est pas soumis à variation par un paradigme différencié ;

- il n'appartient pas à un arrière-plan de la signification, mais à un avant-plan puisqu'il contient la tête logique de la définition (du moins si l'on accepte de concevoir la définition comme un énoncé logique).

- il est une fraction clairement identifiée du signifié référentiel et accessible à l'analyse.

31 Si le $\mathrm{N}$ appartient à une strate supérieure (s'il est lui-même hyperonyme ou superordonné) ses propres hyperonymes et superordonnés (s'il en existe) viendront instancier cette fraction du signifié, mais également ses subordonnés prototypiques. Ces derniers sont, sur le plan catégoriel, des dénominations préférées. Ainsi dans le paradigme des subordonnés de oiseau, moineau ou pigeon instancieront davantage le signifié topique de leur superordonné que casoar, poule, kiwi ou autruche. Ceci implique que ce signifié topique contient des informations relatives notamment au mode de déplacement (« vol »), à la dimension (« petit »), aux sites habités (« urbains : villes, parcs, jardins »), à la relation à l'homme (« non-domestique », « non-sauvage »).

À la différence de ce que l'on observe pour les $\mathrm{N}$ disposant d'un paradigme de reformulants familiers comme tête ou des $\mathrm{N}$ standards subordonnés, ces $\mathrm{N}$ présentent un signifié topique :

- non-vectorialisé: les instructions ne sont indexées que dans la reformulation par un subordonné. Leur instanciation peut donner lieu à des variations selon la nature du prototype. Que celui de l'oiseau change diachroniquement ou varie synchroniquement, la modalisation évoluera avec;

- inscrit dans un arrière-plan de la signification référentielle car non-instanciables au niveau de hiérarchie concerné par le $\mathrm{N}$. Il est impossible de définir les dimensions d'un oiseau, son habitat, son mode de déplacement, ses relations à l'homme, toutes propriétés qui ne sont modalisables qu'au niveau des espèces subordonnées ;

- dont la modalisation est inscrite dans un univers de croyance aux tenants prototypiques. La modalisation / vectorialisation instanciée par moineau ou pigeon relativement à oiseau ne tient sa validité que de la position prototypique de ces deux N, contrairement à ce que l'on observe pour tête, qui dispose d'un paradigme de coréférents cohyponymes attestés lexicalement, donc stables et déconnectés d'une détermination psychologique.

Pour résumer, le sens d'une UL est externalisé, exprimé dans un ailleurs :

- dans le cas d'un N FAM il est localisé dans le signifié mémoriel ;

- pour le $\mathrm{N}$ standard il est localisé dans le signifié topique, fraction émergente et saillante du signifié référentiel. Il est également véhiculé et (partiellement) vectorialisé par le signifié mémoriel d'un reformulant (familier).

L'existence d'une hybridation interne conditionne le statut d'UL: nous contestons l'hypothèse selon laquelle le lexique est partagé en UL prototypiques (les $\mathrm{N}$ standards) opposées à d'autres moins représentatives (les N FAM) qui ne tiendraient leur sémiotique d'UL que par procuration. Nous lui préférons celle selon laquelle il est structuré en UL nucléaires (les $\mathrm{N}$ standards) qui voient leur signifié topique exprimé, et en UL externalisantes (les reformulants, familiers ou non) qui externalisent le signifié topique des précédentes. Toute unité standard peut cumuler une fonction d'externalisation si elle 
est susceptible d'intervenir comme reformulant. En revanche certains N, notamment les $\mathrm{N}$ FAM, du fait de leur prédisposition à la reformulation d'une donnée originée dans le standard, ne sauraient être que des items externalisants.

L'hybridation interne, même observée ici superficiellement, affecte l'ensemble du lexique quel que soit son registre d'emploi. C'est dire que la sémiotique de l'UL est intrinsèquement écartelée et non pas unifiée par un différentiel (hypothèse structurale) ou une positivité référentielle (hypothèse prototypique). Cette hybridation est linguistique (et non pas pragmatique) parce qu'inscrite dans le lexique, hors contexte, et repérable par lui sans recours à une situation d'énonciation. Elle est également le moteur d'une dynamique de l'UL qui ne saurait penser son identité que dans l'ouverture, le déplacement.

\section{L'hybridation externe et la question de la dénomination}

36 La dénomination, telle qu'elle a été conceptualisée par Kleiber (1984), est une propriété sémiotique des noms qui consigne leur statut d'unité lexicale. Dénommer, c'est référer en utilisant un item qui répond à la question Comment s'appelle ce $x$ ? Quel est le nom de ce $x$ ? L'UL qui satisfait à ce test sera considérée comme une dénomination, p.ex. tête, voiture, citron ( " fruit »), caisse (" grande boîte, coffre »).

37 La dénomination s'oppose à la désignation, qui est un mode de référenciation occurrentiel construit par le locuteur et non par la langue. Ainsi référer à des fauteuils en utilisant l'expression commodités de la conversation ou à un restaurant d'entreprise par palais de la gastronomie revient à les désigner (et non pas les dénommer).

La dénomination est une spécificité du statut d'unité lexicale (Mortureux 1997). Elle synthétise l'idéal du signe saussurien lequel dans sa fonction référentielle ne saurait être que dénominatif. Une telle conception présente un certain nombre de difficultés dont nous avons fait état dans Petit (1999). Elle n'en reste pas moins, par son caractère totalisant et sa validation binaire ${ }^{6}$ un révélateur efficace de l'hybridation externe de l'UL.

\section{a. l'incomplétude de la fonction dénominative}

L'exemplification choisie par Kleiber et l'argumentation fournie à l'appui de sa démonstration satisfont tout à fait l'intuition linguistique puisqu'il est vrai que :

- une petite mouche s'appelle un moucheron

- moucheron est le nom d'une petite mouche

et que ce type de test est celui qui par définition convient à l'identification de la fonction référentielle d'un $\mathrm{N}$.

De fait il est possible d'effectuer une discrimination, au sein du lexique entre UL dénominative et UL non-dénominative :

- la partie supérieure du corps de l'homme [...] s'appelle la tête

- tête est le nom de la partie supérieure du corps de l'homme

- *la partie supérieure du corps de l'homme [...] s'appelle (la /le) caboche, ciboulot, citron cigare, carafe, caillou, cassis, cloche

- *aboche, ciboulot, citron cigare, carafe, caillou, cassis, cloche est le nom de la partie supérieure du corps de l'homme 
41 Le rejet se justifie pour ces derniers par le fait qu'ils réfèrent à travers un univers de croyance (Kleiber et Tamba 1990). On en déduira que :

- les N FAM sont incapables de dénommer

- la possession du statut dénominatif est lié au type de propriétés, en l'occurrence descriptives, instanciées par le $\mathrm{N}$ lors de la visée référentielle

- les N FAM ne sont pas pleinement des UL

L'incapacité supposée des N FAM à dénommer peut s'expliquer si l'on admet qu'ils «servent à pointer des réalités dont ils ne sont pas le nom (name) " (Cadiot 1997). Leur relation référentielle est inscrite en langue sans qu'ils l'assument au même titre qu'une dénomination statutaire. De fait citron, citrouille, carafe, cafetière dans leur valeur " tête " seraient, d'après Cadiot, des emplois non-dénominatifs (de $\mathrm{N}$ dénominatifs). Ciboulot, caboche, clebs, etc., qui sont monosémiques, constitueraient des $\mathrm{N}$ non-dénominatifs.

La raison principale de cette incapacité proviendrait de ce que, contrairement à tête ou chien, ils recouvrent des catégories référentielles de dicto et non pas des catégories de re. La prédication tenue par le signifié mémoriel sur le monde joue indiscutablement un rôle dans la construction de la catégorie dans la mesure où, s'il dresse une figuration du référent, il conduit a priori le signe à opérer une visée indirecte, médiatisée par un univers de croyance.

Plusieurs objections peuvent être faites à cette conception. D'abord rappelons, une fois de plus, que la valeur référentielle des N FAM précités est inscrite dans le lexique. Ceci implique qu'ils possèdent en commun avec les $\mathrm{N}$ standards dénominatifs :

- la construction de la catégorie référentielle. Le référent associé à caboche, citron, etc. est identique extensionnellement à celui de tête. En ceci ils catégorisent effectivement des individus. Pour crinière, poubelle («voiture mal entretenue ») ou caserne («grand immeuble peu plaisant ») la référence est inédite du fait que les réalités couvertes ne disposent pas de dénominations en standard. Elle est constituée en sous-classe de celle du N standard qui fait fonction pour eux d'hyperonyme ;

- le processus d'acquisition de la valeur référentielle: mémorisation de la relation, apprentissage, assignation de la référence impossible depuis une analyse du signifiant ;

- le mode de référenciation: codage de la relation référentielle, instituée soit par acte de baptême soit par habitude associative ;

- le mode de donation du référent : préconstruit culturel originé dans la langue (et non pas construction discursive).

Ces éléments induisent qu'en tout état de cause les N FAM ne peuvent pas être des désignations mais sont apparentées sémiotiquement aux dénominations. Elles en possèdent le mode de fonctionnement mais le statut ne leur est pas reconnu. Le refus ne s'explique pas par des raisons linguistiques mais par l'impensé théorique qui fournit l'arrière plan à la conception traditionnelle de l'UL. Entre autres, le fait que le privilège soit accordé au registre standard, masquant ainsi l'hétérogénéité du lexique, et par là même l'existence d'une polydénomination (dénomination simultanée par des items diversement localisés dans le lexique).

Ce privilège fonde les conditions juridiques et non pas linguistiques de la dénomination. celle-ci, telle qu'elle est pensée depuis Kleiber (1984) n'est pas tant une propriété lexicale qu'une une imputation culturelle. Ainsi si l'on rejette les énoncés précités (*la partie supérieure du corps de l'homme [...] s'appelle (la /le) caboche, ciboulot, citron cigare, carafe, caillou, cassis, cloche; *aboche, ciboulot, citron cigare, carafe, caillou, cassis, cloche 
est le nom de la partie supérieure du corps de l'homme) c'est parce que l'on refuse de penser une péréquation des registres qui les validerait en spécifiant :

- X est le nom standard d'un $\mathrm{x} / \mathrm{un} \mathrm{x}$ s'appelle en registre standard un $\mathrm{X}$

- $X$ est le nom familier d'un $\mathrm{x} /$ un $\mathrm{x}$ s'appelle en registre familier un $\mathrm{X}$

Deux conséquences immédiates sont à tirer si l'on admet cette péréquation : le registre standard ne serait plus confondu avec le lexique mais apparaitrait dans sa véritable nature d'option adaptée à un type d'échanges spécifique. Ensuite, du fait de la multiplicité des reformulations attestées en FAM pour une même donnée référentielle (cf. l'exemple de la tête) on admettra également l'existence d'une polydénomination de second rang qui s'exprimera ainsi :

- caboche, ciboulot, citron cigare, carafe, caillou, cassis, cloche sont les noms familiers de la partie supérieure du corps de l'homme- caboche est l'un des noms familiers de la partie supérieure du corps de l'homme

e cas de crinière et poubelle est quelque peu différent. Si l'on peut désormais admettre :

- crinière / poubelle est l'un des noms familiers d'un $\mathrm{x}$

on ne peut pas pour autant dire que le x dispose d'une dénomination standard. Dans ce cas les items FAM sont les seuls dénommants. La réalité à laquelle ils renvoient leur est spécifique.

On nous objectera que tête et citron ne construisent pas leur catégorie référentielle de manière identique. On retrouve ici la distinction de re / de dicto. Il est vrai qu'une partie du lexique standard (notamment celui sélectionné pour cette étude : tête, voiture, chevelure , immeuble...) réfère à des catégories fondées sur la possession de propriétés critériales, objectives, intrinsèques ou extrinsèques mais toujours descriptives (nous les appellerons propriétés $W$ ). Le vocabulaire familier tend à se comporter à cet égard de manière hybride. Le signifié référentiel d'un N FAM effectue sa visée par l'intermédiaire des mêmes propriétés que celles instanciées par le signifié référentiel du $\mathrm{N}$ standard qui lui correspond $^{7}$ (ce que confirme l'analyse des définitions lexicographiques). En revanche, le signifié mémoriel, du fait qu'il produit une figuration trivialisante, réalise des propriétés appréciatives (que nous appellerons propriétés $Z$ ). Le signifié référentiel construit et régule l'extensité du N FAM et son intension dénotative. Le signifié mémoriel construit son intension figurative. Or c'est ce dernier précisément qui fonde la perception de dicto de la catégorie référentielle construite par le N. Si tête dénomme une catégorie de re, ciboulot, citron, caboche dénomment quant à eux des catégories paradoxales : de dicto sur le plan intensionnel du fait que la figuration résume une prédication appréciative, mais de re sur le plan extensionnel (elles sont préconstruites et codées puisque déjà dénommées en registre standard).

\section{b. les propriétés $Z$ et la dénomination en registre standard}

51 Les propriétés $Z$ véhiculent un contenu appréciatif et fondent, dans le cas des N FAM, la figuration du référent par le signifié mémoriel. Elles peuvent être explicites ou implicites. Elles sont explicites dans la définition de $\mathrm{N}$ comme crinière, caserne ou poubelle qui isolent une sous-catégorie à l'intérieur de celle du $\mathrm{N}$ standard auquel ils sont reliés (nous soulignons) :

- chevelure abondante- grand immeuble peu plaisant- voiture mal ou non entretenue 

les trois $\mathrm{N}$ précités) du fait que celui-ci opère une conceptualisation trivialisante du réel. En ceci elles participent crucialement à la sémiotique de ce vocabulaire en fondant la familiarité comme proximité référentielle désacralisante. également dans la sémiotique des items standards évaluatifs : spécialiste, expert, avarice, beauté, amélioration, imprudence... Si les propriétés $\mathrm{W}$ fondent des dénominations (des noms catégorisants), les propriétés $Z$ définissent, selon Cadiot, les noms typants c'est-àdire des $\mathrm{N}$ qui «servent de principe de regroupement des $\mathrm{x}$ dont on dit (pense, croit, imagine) qu'ils ont la propriété $\mathrm{X}$ [...] ils ne supposent aucune condition a priori pour la validation de leur emploi ». fait qu'ils effectuent leur visée référentielle à travers un univers de croyance et de ce fait isolent des catégories non préconstruites et non codées. Cette particularité semble opérer comme principe invalidant: toute UL instanciant $\mathrm{Z}$ dans son signifié référentiel ou mémoriel est jugée de facto incapable de dénommer. La raison impliquée usuellement est que son référent n'est pas défini préalablement par la possession d'attributs critériaux c'est-à-dire de propriétés descriptives, d'où le sentiment qu'il ne suppose pas de condition a priori pour la validation de l'emploi du $\mathrm{N}$.

Sans pour autant remettre en cause cette analyse, nous contesterons sa conclusion sur la valeur dénominative des $\mathrm{N}$ référant par $\mathrm{Z}$. La différence entre voiture et bagnole ou achat et imprudence (si l'on considère que tel achat a été une imprudence) réside dans le fait que l'attribut critérial, car les $\mathrm{N}$ dotés de $\mathrm{Z}$ réfèrent bien sur la base d'attributs critériaux (voir plus bas), n'est pas une PI mais une PE non-objectale du fait qu'elle est inscrite dans un univers de croyance, lexicalisée et prévue par le programme sémantique de l'unité.

es $N$ qui visent leur référent a travers $W$ sont des dénominations catégorielles. Ils n'opèrent pas de figuration et effectuent une conceptualisation aseptisée (a priori objective, puisque non médiatisée par un univers de croyance) dont le centre de gravité est fourni par la structure du référent et sa fonctionnalité. Nous admettrons que les $\mathrm{N}$ standards qui réfèrent par l'intermédiaire de $\mathrm{Z}$ sont également des dénominations. Leur sémiotique se fonde structurellement sur le même principe que les précédents. Si l'on peut dire qu'achat ou voiture sont respectivement le nom d'un $\mathrm{x}$ qui $\mathrm{W}$ (et qu'un $\mathrm{x}$ qui $\mathrm{W}$ s'appelle un achat / une voiture), on admettra qu'imprudence, escroquerie, lâcheté, expert ou spécialiste :

- est le nom d'un x qui Z- un x qui Z s'appelle un(e) imprudence / escroquerie /

lâcheté / expert / spécialiste

Les $\mathrm{N}$ appréciatifs se distinguent des descriptifs en ce que les différences spécifiques incluses dans leur définition logique sont modalisantes. L'incluant pour sa part est représentable par un hyperonyme (imprudence, lâcheté: « conduite, action qui consiste à $\mathrm{Z}$; le résultat de cette conduite, de cette action »). Ces $\mathrm{N}$ s'inscrivent dans des hiérarchies lexicales en circonscrivant à l'intérieur d'une catégorie préconstruite et codée (celle de l'hyperonyme ou du superordonné) un espace de non-stabilisation (ils ne fondent pas une catégorie codée). C'est ce qui les différencie de leurs antagonistes par W, qui lexicalement emboîtent des espaces référentiels stabilisés et assurent l'homogénéité (l'absence de disruption sémiotique) des hiérarchies lexicales. 
Dans Petit (1999), nous avons proposé de considérer les $\mathrm{N}$ qui réfèrent au travers de propriétés $\mathrm{Z}$ comme des dénominations occurrentielles. Dénominations du fait que :

- la maîtrise de ces $\mathrm{N}$ nécessite un apprentissage et une mémorisation, même réduits ;

- la relation référentielle qu'ils couvrent est inscrite dans le lexique, même si elle s'applique à une catégorie ouverte ;

- pour être référé par ces $\mathrm{N}$ le $\mathrm{x}$ doit impérativement satisfaire à une condition a priori $(\mathrm{z})$ bien que celle-ci, en tant qu'appréciation, soit en principe incapable de circonscrire une catégorie objectale.

Occurrentielles parce que transverses. Les $\mathrm{x}$ qui sont susceptibles de les investir possèdent déjà une dénomination catégorielle (objectale) par $\mathrm{W}$ et peuvent appartenir à des catégories référentielles distinctes définies par $\mathrm{W}$. La dénomination par $\mathrm{Z}$ est toujours susceptible de réfutation sans que cette dernière génère une décidabilité quant au référent. Sauf exemplaires totalement atypiques, il est encore possible de décider ${ }^{8}$ entre p.ex. une voiture ou une camionnette, et donc de justifier l'emploi de tel $\mathrm{N}$ pour assurer la référenciation. En revanche, pour les appréciatifs standards l'assignation de nom n'est pas légitimée par la nature de re du référent (- C'est de la lâcheté. - Pas du tout, c'est de la prudence / sagesse / fidélité).

Les $\mathrm{N}$ instanciant $\mathrm{Z}$ dénomment une facette du référent (une conceptualisation possible). S'ils appartiennent au registre familier cette dénomination est perçue comme inscrite dans un paradigme alterne. Toutefois elle ne l'est réellement que dans le cas où les $\mathrm{N}$ concernés appartiennent à des binômes situés sur plus d'un registre (voiture standard / bagnole FAM), c'est-à-dire dans les cas de polydénomination.

61 Si l'on admet, comme nous le faisons, que lexicalement les $\mathrm{N}$ contenant une propriété $\mathrm{Z}$ sont des dénominations, on vide par contrecoup la désignation de sa substance du moins sur le plan lexical. Sauf à considérer que la désignation est une modalité d'actualisation de la référence et qu'elle ne ressortit pas au lexique (même si elle peut être lexématique, Petit 1995) mais au discours, à la mise en verbe. L'espace de cet article ne nous permet pas de traiter la question. Nous exposerons ici quelques axes. Il y a désignation dans le cadre de la référence discursive assurée par un $\mathrm{N}$ n'instanciant que $\mathrm{W}$ :

- quand ce $\mathrm{N}$ opère une modification de la focalisation, notamment un élargissement (un chien ,... cet animal) ;

- quand il est inclus dans un $\mathrm{SN}$ où les déterminations qui l'accompagnent opèrent une modification de sa focalisation intrinsèque. Dans magasin où l'on vend des livres et ancien secrétaire général de la préfecture de la Gironde, magasin et secrétaire général sont des dénominations. Toutefois ils se voient déqualifiés par leur environnement lexicosyntaxique qui restreint le champ de la focalisation respectivement sur un type de magasin et sur un titre paramétré dans le temps, l'espace et l'institution administrative.

Egalement lorsque cette référence est assurée par un $\mathrm{N}$ instanciant $\mathrm{Z}$ :

- du fait qu'il est en emploi qualifiant (Cadiot 1997) : ton fonctionnaire de père

- s'il est en position anaphorique relativement un $\mathrm{N}$ visant par $\mathrm{W}$ et susceptible de satisfaire aux tests de dénomination (ton bureau,...ce capharnaüm)

- non lexicalisé dans son emploi référentiel (en parlant de la délinquance juvénile : ...les fauves sont lâchés dans les rues sans dresseur pour les dompter ${ }^{9}$ (nous soulignons)).

Pour que la question de la désignation ait une pertinence, il convient que celle de la dénomination soit résolue en discours, mais aussi en amont dans le lexique. Les exemples sélectionnés traditionnellement dissipent toute équivoque (librairie, pomme de terre, boîte, 
client...). Ils s'inspirent d'une conception adamique de la dénomination (un nom pour chaque chose, chaque chose a son nom) confirmant ainsi l'homogénéité souhaitée dans la représentation du lexique.

L'observation du matériau lexical et discursif démontre qu'au moins trois types de perturbations viennent troubler cet ordonnancement. Nous les exposerons ici très brièvement. Elles concernent :

- l'assignation de référent, c'est-à-dire l'identification de la catégorie visée par un item lexical. La question se pose ordinairement pour la néologie. Elle peut intéresser également le lexique dans son utilisation la plus courante. Ainsi, sauf à avoir recours à une définition terminologique, une indistinction existe entre les stéréotypes culturels de certains $\mathrm{N}$ cohyponymes comme tube / tuyau ou maison / immeuble. Au point que si leur valeur dénominative ne saurait être mise en doute il semble difficile d'indiquer précisément de quoi ils sont les dénominations;

- l'assignation de nom. Est concernée la distinction entre ce que nous avons appelé (Petit 1998b et 1999) dénomination de droit et dénomination de fait. Un garde forestier, dénomination de fait, s'appelle officiellement un agent technique forestier, dénomination de droit (France Info 27/07/98). La dénomination chlorofluorocarbone massivement utilisée par la presse (dénomination de fait) pour référer aux gaz CFC est fautive aux yeux des spécialistes - ce $\mathrm{N}$ dénommant faussement son référent - contrairement à chlorofluorocarbure (dénomination de droit), que les dictionnaires ont finalement enregistrée en remplacement de la précédente (dénomination de droit s'imposant comme dénomination de fait). Klaxon, Frigidaire, Hygiaphone, Abribus sont considérés et utilisés en toute illégalité par la communauté linguistique comme des lexèmes (dénominations de fait s'imposant en contravention avec des dénominations de droit);

- l'assignation de statut sémiotique. Les expressions procureur indépendant ou pastille verte ${ }^{10}$ dénomment-elles ou bien sont-elles des désignations ? Si la première correspond à un titre ( procureur indépendant vs procureur X) elle est une dénomination, sinon elle fournit (Petit 1998b) une pseudo-dénomination (séquence utilisée de manière stable et récurrente par un groupe pour la prise en charge d'une relation référentielle, en marge d'une dénomination déjà instituée). Pastille verte est actuellement un néologisme en phase avancée de lexicalisation. Il est répandu dans le discours courant et figure sur le référent comme identifiant.

\section{En guise de conclusion}

L'espace de cet article ne permet pas de produire une conclusion sur les phénomènes observés. On peut néanmoins dégager deux acquis, que la recherche devrait affiner :

- le statut d'UL est traditionnellement considéré comme un label, décerné par le linguiste, et qui a pour fonction de consigner la stabilisation / fossilisation de propriétés sémantiques et sémiotiques. La stratification constatée du signifié vient introduire du bougé dans la représentation. Elle ouvre l'UL sur son extérieur sans pour autant tomber dans le fixisme d'une perspective systémique. L'UL entretient une relation dynamique au lexique. Du fait qu'elle est l'enjeu d'une investigation lexicale du sens (par le biais de la reformulation), cette relation constitue un moyen, peut-être le seul, d'aboutir à une détermination (lexicale) du sens (lexical);

- le statut d'UL est traditionnellement tautologique. Il est appliqué à un type de matériau idoine, particulièrement adapté à la spécificité qu'il a pour charge de consigner. L'hypothèse 
de l'hybridation sémiotique est étrangère à cette conceptualisation. Elle permet d'intégrer et de rendre compte du fonctionnement d'unités telles que les noms familiers, qui sont usuellement reléguées dans les coulisses de la lexicologie. De fait une représentation globale devient possible, non plus en termes de prééminence juridique (certains items seraient de véritables UL, d'autres ne le seraient que par délégation ou tolérance), mais de fonctionnalité sémiotique : une UL est une unité de dénomination définie par sa fonction nucléaire ou externalisante.

\section{BIBLIOGRAPHIE}

ANSCOMBRE J.-Cl. 1995 La théorie des topoï, Kimé

CADIOT P 1997 « Aux sources de la polysémie lexicale », Langue française n¹17, Larousse, Paris

CADIOT P et NEMO F. 1997 « Pour une sémiogenèse du nom », Langue française n¹13, Larousse, Paris

CADIOT P et NEMO F. 1998 « On n'a pas toujours la tête sur les épaules», Sémiotiques nº 13

FRANCKEL J.-J. et LEBAUD D. 1992 « Lexique et opérations. Le lit de l'arbitraire », La théorie d'Antoine Culioli, Ophrys

FRANCKEL J.-J., PAILLARD D. et SAUNIER E. 1997 « Modes de régulation de la variation sémantique d'une unité lexicale. Le cas du verbe passer ", La locution : entre lexique, syntaxe et pragmatique, Klincksieck

GRIZE J.-B. 1990 Logique et langage, Ophrys

GRUNIG B.-N. 1982 « Différences et ajustements », Langue française n53, Larousse

GRUNIG B.-N. et R. 1982 La fuite du sens Credif, Hatier

KLEIBER G. 1984 « Dénomination et relations dénominatives », Langages n76, Larousse

KLEIBER G. 1990 La sémantique du prototype, PUF

KLEIBER G. et Tamba I. 1990 «L'hyponymie revisitée : inclusion et hiérarchie », Langages n98, Larousse

MARTIN R. 1976 Inférences, antonymie et paraphrase, Klincksieck

MEL'CUK 1988 « Paraphrase et lexique dans la théorie Sens-Texte », Lexique n6, Presses universitaires de Lille

MORTUREUX M.-Fr. 1997 La lexicologie, entre langue et discours, Sedes

NICOLAS C. 1995 «Je suis omnibulé par ma rénumération » : quelques notes sur le phénomène de remotivation lexicale par attraction panonymique, Les cahiers de lexicologie, $n^{\circ} \mathrm{LXVI}$, Didier

PETIT G. 1995 « La désignation de timbre-poste », Les carnets du CEDISCOR n³, Presses de la Sorbonne Nouvelle, Paris

PETIT G. 1998a « Un phénomène d'hybridation sémiotique et sémantique : les noms familiers », Le français moderne LXVI- ${ }^{\circ} 1$, CILF 
PETIT G. 1998b « Dénomination et discours » Actes du 1er Symposium international d'analyse du discours, Universitad complutense de Madrid

PETIT G. 1999 « Dénomination et lexique » (à paraître) Journal of french language studies, Cambridge University Press.

SIBLOT P. 1997 « D’un nom l'autre », Nouvelle revue d'onomastique n²9-30

SiвLOT P. 1998 « Signifiance du praxème nominal », L'information grammaticale n77

VARGAS LLOSA M. 1997 Les enjeux de la liberté, Gallimard, Paris

\section{NOTES}

1. Exception faite pour la praxématique (Siblot, notamment 1997, 1998), qui toutefois pose crucialement la question, non clairement élucidée, de l'existence même du lexique

2. Nous entendrons par noms familiers des items étiquetés comme tels par les principaux dictionnaires de langue et dont l'appartenance à ce registre est reconnue par la communauté des locuteurs. Il peut s'agir de $\mathrm{N}$ exclusivement familiers (clebs, salaud) ou de $\mathrm{N}$ disposant par ailleurs d'un emploi standard (caillou, cassis : « tête, crâne »).

3. La distance catégorielle entre les référents est une condition de la familiarité

4. Parce qu'il se fonde dans son principe sur une logique de topoï, de vérités générales implicites. Toutefois à la différence d'un topos ou d'un stéréotype, la lexicalisation des instructions sémantiques implique une sédimentation de celui-ci dans la langue et non dans la croyance sociale, même si une croyance est à l'origine de sa constitution.

5. Une représentation par CNS du N lit ne produira pas le même listage qu'une perspective prototypique, ni qu'une modélisation culiolienne ou encore une approche en logique naturelle ou en praxématique

6. Totalisant car la conception de Kleiber, qui ne fait que rejoindre la conceptualisation traditionnelle de l'UL, implique que les UL dénomment toutes de la même manière. Binaire car la possession de cette propriété s'opère selon la logique digitale : une unité est dénominative ou ne l'est pas

7. Bien que pour certains items (crinière, caserne, poubelle) il ajoute des spécifications

8. Nous nous situons hors contexte, mais la décision peut être emportée par la possession d'une gestalt et / ou la satisfaction d'attributs critériaux dans une définition lexicale ou terminologique 9. M.Vargas Llosa (1997 : 379)

10. La première a été utilisée de manière quasi exclusive par la presse pour référer au procureur américain qui instruit l'affaire Lewinsky / Clinton, la seconde renvoie à un autocollant signalant les voitures dites non-polluantes

\section{RÉSUMÉS}

Le statut d'unité lexicale (UL), tel qu'il est traditionnellement pensé par la lexicologie, produit une schématisation monolithique de son objet. Les modèles de description classiques, mais également les alternatives récentes sont lestées par le présupposé que la structure sémantique et 
référentielle de l'UL est intrinsèquement homogène. Nous proposons à l'inverse de considérer l'hybridation comme donnée constitutive du statut d'UL : hybridation interne par la stratification du signifié en une composante référentielle ou mémorielle, et ne composante topique; hybridation externe par le clivage dont la relation dénominative fait l'objet entre référenciation descriptive et référentiation appréciative.

The status of lexical item, by the way it is traditionnaly conceptualized in lexicology, produces a monolithic schematization of its object. The classical and more recents patterns of description are founded upon the presupposition that the semantic and referntial structure of lexical item is intrinsically homogeneous. In opposition with this we propose to consider hybridity as a basic principle of the lexical status: internal hybridity due to stratification of the content in a referential and a topic component; external hybridity due to stratification of denominative relation between descriptive and valuative modes to refering.

\section{AUTEUR \\ GÉRARD PETIT}

Université de Paris X - Nanterre 